\title{
Multiple plasma proteins control atrial natriuretic peptide (ANP) aggregation
}

\author{
C Torricelli, E Capurro, A Santucci' ${ }^{1}$ A Paffetti ${ }^{1}$, C D'Ambrosio' ${ }^{2}$ A Scaloni², \\ E Maioli and A Pacini \\ Department of Physiology, University of Siena, via Aldo Moro, 53100 Siena, Italy \\ ${ }^{1}$ Department of Molecular Biology, University of Siena, via Fiorentina 1, 53100 Siena, Italy \\ 2Proteomics and Mass Spectrometry Laboratory, ISPAAM, National Research Council, via Argine 1085, 80147 Napoli, Italy
}

(Requests for offprints should be addressed to A Pacini, Department of Physiology, Via Aldo Moro-53100 Siena, Italy; Email: pacinia@unisi.it)

\begin{abstract}
We have recently demonstrated that human $\alpha$-atrial natriuretic peptide ( $\alpha$-hANP), an amyloidogenic peptide responsible for isolated atrial amyloidosis, binds to a dimeric form of apo A-I belonging to small high-density lipoproteins (HDL). This binding phenomenon is considered a protective mechanism since it inhibits or strongly reduces the ANP aggregation process. The observation that plasma exhibits at least four times greater amyloid inhibitory activity than HDL prompted us to determine whether small HDL are the only ANP plasma-binding factors. After incubation of whole plasma with labelled ANP, the macromolecular complexes were subjected to two-dimensional gel electrophoresis followed by autoradiography. The results presented here provide novel evidence of additional binding proteins, in addition to apo A-I dimer, able to bind ANP in vitro and to prevent its aggregation. The mass spectrometry analysis of the radioactive spots identified them as albumin, $\alpha-1$ antitrypsin, orosomucoid and apo A-IV-TTR complex. The putative impact of these findings in the amyloidogenic/antiamyloidogenic peptides network is discussed.
\end{abstract}

Journal of Molecular Endocrinology (2004) 33, 335-341

\section{Introduction}

The term 'amyloidosis' refers to a group of disease processes in which normally soluble proteins polymerize to form insoluble fibrils. The amyloid fibrils associate with extracellular matrix proteins and proteoglycans to form amyloid deposits which invade the extracellular spaces of organs destroying normal tissue architecture and function (Tan \& Pepys 1994, Merlini \& Bellotti 2003).

Amyloid deposits in the ageing heart are a common finding at autopsy (McCarthy \& Kasper 1998); in the majority of cases, it is an age-related cardiac amyloidosis that can be separated into two groups. The first one is a systemic form, known as senile systemic amyloidosis (SSA) (Sletten et al. 1980, Pitkanen et al. 1984), in which the protein fibril is derived from transthyretin (TTR); SSA mainly affects cardiac ventricles, although other organs are also involved. The other form is isolated atrial amyloid (IAA) (Takahashi et al. 1998). The precursor protein is atrial natriuretic peptide (ANP), a hormone synthesized by the cardiac atria, controlling salt and water homeostasis Johansson \& Westermark 1990). Amyloid deposits are localized at the cardiac atria. This condition is highly prevalent in elderly people. Although the clinical importance of IAA is still poorly defined, several studies have shown that significantly raised plasma levels of ANP may be related to the increased incidence of cardiac pathological conditions, rather than resulting from ageing alone. In fact, patients with cardiac insufficiency, a condition characterized by elevated concentrations of ANP, have an increased prevalence of IAA (Looi 1993). As amyloid deposits are localized at the site of ANP synthesis, cardiac diseases that increase the synthesis and secretion of peptide may account for high local concentrations and consequently affect the rate of formation, progression and consequences of IAA (Rocken et al. 2002). 
It is well known that intact ANP alone is capable of forming amyloid fibrils when incubated in vitro at microgram level (Johansson et al. 1987). In a previous work (Maioli et al. 2000), we observed, in plasma of normal subjects, a lack of aggregated ANP. We hypothesized at that time that the plasma ANP levels under normal conditions are too low to reach the concentration threshold necessary to generate a nucleus. As for other amyloid-forming peptides, ANP polymerization is a nucleationdependent process (Jarrett et al. 1993). An ANP nucleus in vivo can be locally generated whenever peptide accumulates in sufficient amount in extracellular space, and a preformed nucleus is often present in commercial preparations of the synthetic peptide. In fact, we demonstrated that ANP has a great propensity to self-aggregation in vitro even at physiological concentration (pg). Incubation of $\alpha-{ }^{125} \mathrm{I}-\mathrm{hANP}$ in PBS at $37{ }^{\circ} \mathrm{C}$ for $24 \mathrm{~h}$ led to the formation of a macromolecular form consisting of self-aggregated ANP. Moreover, the addition of plasma proteins to the incubation mixture gave rise, in addition to aggregated form, to ANP-protein complexes; in this case, the amount of aggregated ANP was strongly decreased. From this observation, we suggested that plasmabinding factors, by sequestering ANP, affect the rate of amyloid formation by delaying or inhibiting the aggregation process (Maioli et al. 2000).

More recently (Torricelli et al. 2004), we showed that high-density lipoproteins (HDL) are involved in the sequestration phenomenon. In particular, we suggested that ANP binds to an apo A-I dimeric form which probably belongs to small HDL. HDL are generally considered to protect against amyloidosis by inhibiting or strongly reducing the formation of amyloid fibrils. In fact, protein constituents of HDL have been shown to bind and form complexes with other well-known amyloidogenic peptides, such as amyloid $\beta$-peptide (A $\beta$ ) (Koudinov et al. 1994, 1998) and TTR (Sousa et al. 2000). Moreover, particularly with regard to $A \beta$, some researchers have identified 13 plasma proteins that, at physiological concentrations, are potent inhibitors of $\mathrm{A} \beta$ polymerization (Bohrmann et al. 1999).

The starting point of the present work was the finding that plasma exhibits at least four times greater amyloid inhibitory activity than HDL. This result prompted us to determine whether small HDL are the only ANP plasma-binding factors.
Here, we present novel evidence for the existence of additional endogenous factors able to bind ANP and to prevent its aggregation. This finding is of great importance, since the current treatments of amyloidoses are based mainly on decreasing the availability of the amyloid protein precursor or on blocking conformational changes of the amyloid protein (Lachmann \& Hawkins 2003). The identification of natural protective mechanisms could open a new area of research in the development of new therapeutic strategies to prevent and treat these debilitating diseases.

\section{Materials and methods}

\section{Materials}

Equipment and all reagents of the highest available grade, unless otherwise indicated, were purchased from Amersham Biosciences. Blood samples obtained from healthy volunteers were collected into EDTA and aprotinin $(500 \mathrm{kIU})$ and immediately centrifuged at $1500 \boldsymbol{g}$ for $15 \mathrm{~min}$ at $4{ }^{\circ} \mathrm{C}$. Aliquots of pooled plasma were stored at $-70{ }^{\circ} \mathrm{C}$ until the time of use.

\section{Ligand binding of $\alpha^{-125}$-hANP to HDL or whole plasma}

Lyophilized $\alpha-{ }^{125} \mathrm{I}-\mathrm{hANP}$ (specific activity 2000 $\mathrm{Ci} / \mathrm{mmol}$ ) was dissolved in ultrapure water according to the manufacturer's instructions. To evaluate and compare the amyloid inhibitory activity between isolated HDL and whole plasma, physiological concentrations of ANP and an equal amount of commercial HDL (Fluka Sigma-Aldrich, Milan, Italy) or plasma proteins were incubated at $37^{\circ} \mathrm{C}$ for $24 \mathrm{~h}$, as previously reported (Maioli et al. 2001). In a parallel experiment of binding inhibition, plasma proteins were preincubated at $37^{\circ} \mathrm{C}$ for $1 \mathrm{~h}$ with a neutralizing (saturating) amount of polyclonal antibody against apo A-I (Calbiochem; Inalco, Milan, Italy) before incubation of labelled ANP. The separation of free from bound ${ }^{125} \mathrm{I}-\mathrm{ANP}$ (big $\alpha_{-}{ }^{125} \mathrm{I}-\mathrm{hANP}$ ) was achieved by adding a dextran-coated charcoal (DCG) mixture, prepared as reported previously (Maioli et al. 2000). After centrifugation, the supernatant containing big $\alpha_{-}{ }^{125}$ I-hANP was counted and expressed as percentage of total radioactivity added to the incubation mixture. All 
experiments were done in quadruplicate on at least eight independent occasions.

\section{SDS-gradient gel}

Big $\alpha-{ }^{125}$ I-hANP, obtained after incubation of plasma and labelled ANP, was run on a precast continuous gradient SDS-PAGE (4-20\% T, 2.67\% C) (Bio-Rad Laboratories, Milan, Italy) under reducing and non-reducing conditions. The sample was boiled for $5 \mathrm{~min}$ after adding sample buffer containing or not $2 \beta$-mercaptoethanol. Molecular mass standards (14-94 kDa) were run in parallel. Gel was stained for 10 min with $0 \cdot 1 \%$ Coomassie brilliant blue R-250 in 45\% methanol and 10\% glacial acetic acid, destained for $1-2 \mathrm{~h}$ in $10 \%$ methanol and 10\% glacial acetic acid, dried, and exposed for $72 \mathrm{~h}$ at $-70{ }^{\circ} \mathrm{C}$ to Kodak X-Omat AR autoradiography film and a single intensifying screen, as previously reported (Torricelli et al. 2004). The amount of total protein loaded per lane was $50 \mu \mathrm{g}$.

\section{Two-dimensional (2-D)-PAGE under non-reducing conditions}

Big $\alpha^{-125} \mathrm{I}-\mathrm{hANP}$ obtained after incubation of plasma with labelled ANP was subjected to 2-DPAGE. The sample was denatured in the buffer for the first dimension comprising a solution containing $8 \mathrm{M}$ urea, 4\% (w/v) 3-[(3-cholamidopropyl) dimethylammonio]-1-propanesulfonate (CHAPS), $40 \mathrm{mM}$ Tris and a trace of bromophenol blue. 2-D electrophoresis was carried out by procedures detailed elsewhere (Trabalzini et al. 2003). An amount of $1000 \mu \mathrm{g}$ protein sample was applied to an Immobiline strip (IPG: immobilized $\mathrm{pH}$ gradient; Amersham Biosciences, Milano, Italy) consisting of a non-linear gradient, $\mathrm{pH}$ 3.5-10, previously rehydrated. Isoelectric focusing was carried out on Multiphor II (Amersham Biosciences). The voltage was linearly increased from 300 to $3500 \mathrm{~V}$ during the first $3 \mathrm{~h}$ and then stabilized at $5000 \mathrm{~V}$ for $22 \mathrm{~h}$ (total $110 \mathrm{KV} / \mathrm{h}$ ). The IPG strip was then equilibrated in $6 \mathrm{M}$ urea, $30 \%(\mathrm{w} / \mathrm{v})$ glycerol, 2\% (w/v) SDS, 0.05 M Tris-HCl, pH 6.8. Electrophoresis in the second dimension was carried out on a 9-16\% polyacrylamide linear gradient gel $(18 \times 20 \mathrm{~cm} 1.5 \mathrm{~mm})$ at a constant current of $40 \mathrm{~mA}$. The gels were stained for $1 \mathrm{~h}$ with $0 \cdot 1 \%$ Coomassie brilliant blue R-250 in 45\% methanol and $10 \%$ glacial acetic acid, destained overnight in
$10 \%$ methanol and 10\% glacial acetic acid, dried, and exposed for $144 \mathrm{~h}$ at $-70{ }^{\circ} \mathrm{C}$ to Kodak $\mathrm{X}$-Omat AR autoradiography film and a single intensifying screen. Both Coomassie-stained gel and autoradiographic film were subjected to laser densitometry (Molecular Dynamics, Sunnyvale, CA, USA). The digitalized images obtained were compared by Melanie II 2-D-PAGE and PD-Quest softwares (Bio-Rad).

\section{Mass spectrometry}

MALDI-TOF mass spectrometry was used to identify the proteins present in autoradiographic complexes. Spots from 2-D-PAGE were excised from the gel, triturated and washed with water and acetonitrile. Proteins were in-gel reduced, S-alkylated and digested with trypsin as previously reported (Talamo et al. 2003). Peptide mixtures were loaded on the MALDI target, by the dried-droplet technique with $\alpha$-cyano-4-hydroxycinnamic as matrix, and analysed by the Voyager DE-PRO mass spectrometer (Applied Biosystems, Framingham, MA, USA). Internal mass calibration was performed with peptides deriving from trypsin autoproteolysis. The PROWL software package was used to identify spots unambiguously from the National Center for Biotechnology Information and SwissProt nonredundant sequence database (Talamo et al. 2003).

\section{Results}

\section{Ligand binding of $\alpha^{-125}$ I-hANP to HDL or whole plasma}

Pooled plasma samples and HDL were incubated with a physiological amount of labelled ANP. The percentages of radioactivity recovered in the supernatant, after DGG precipitation, were $35 \pm$ $3 \cdot 2 \%$ and $8.9 \pm 2 \cdot 11 \%$ respectively of the total radioactivity added to the incubation mixture. Of note, ANP complexes were about four times higher in plasma than in HDL. When plasma proteins, previously incubated with anti-apo A-I polyclonal antibody, were incubated with labelled ANP, the percentage of radioactivity in the supernatant was unchanged $(34 \cdot 57 \pm 2 \cdot 36 \%)$.

\section{SDS-gradient gel}

Big $\alpha-{ }^{125}$ I-hANP, obtained after incubation of labelled ANP with plasma, preincubated or not 


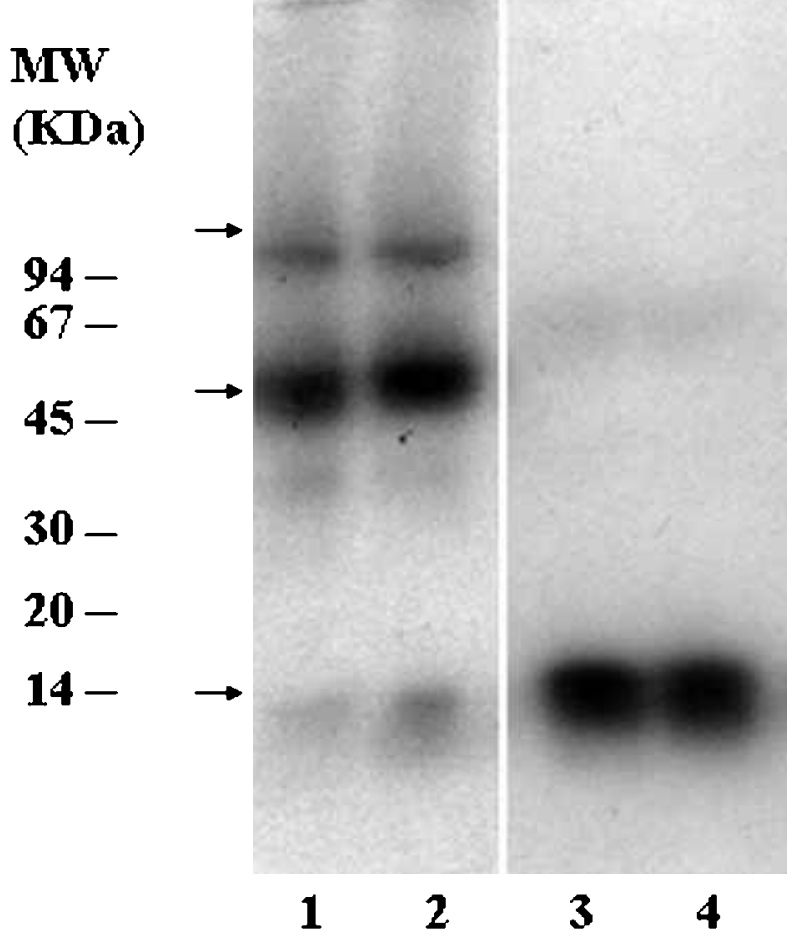

Figure 1 Representative autoradiographical patterns obtained from 4-20\% SDS gel electrophoresis of big $\alpha-\left.\right|^{125}$-hANP. Lanes 1 and 3: big $\alpha-\left.\right|^{125}$-hANP from incubation of $\alpha-\left.\right|^{125}$-hANP and whole plasma was applied on SDS-gradient gel under non-reducing and reducing conditions respectively; lanes 2 and 4: big $\alpha-\left.\right|^{125}$-hANP from incubation of $\alpha-\left.\right|^{125}$-hANP, and whole plasma preincubated with anti-apo A-I polyclonal antibody, was applied on SDS-gradient gel under non-reducing and reducing conditions respectively. The arrows indicate the four radioactive bands (see Results). All experiments were done in duplicate on at least four independent occasions.

with anti-apo A-I, was subjected to electrophoresis on $4-20 \%$ gradient gel under reducing and non-reducing conditions and autoradiography. Gel autoradiography revealed the presence of four radioactive bands when the sample was run under non-reducing conditions (Fig. 1, lane 1): the first band, near the gel origin, represented radioactive material with a molecular mass higher than the resolving capacity of the $4-20 \%$ gradient gel. The second and third bands, the most evident, showed molecular mass greater than $94 \mathrm{kDa}$ in the range $67-45 \mathrm{kDa}$ respectively. The fourth band, visible in the gel dye front, represented free monomeric $\alpha_{-}{ }^{125} \mathrm{I}-\mathrm{hANP}$. The first three bands disappeared in the presence of a reducing agent with a corresponding increase of free monomeric $\alpha-{ }^{125}$ I-hANP (Fig. 1, lane 3). When plasma proteins were blocked with anti-apo A-I antibodies, the same electrophoretic pattern was obtained (Fig. 1, lanes 2 and 4), and no band disappearance was observed. This finding supported the already mentioned ligand-binding results and further strengthened our hypothesis on the existence of several ANP-binding proteins.

\section{2-D-PAGE and protein identification}

Once verified, the stability of the complex towards CHAPS and urea (not shown), big $\alpha-{ }^{125}$ I-hANP, obtained after incubation of plasma and labelled ANP, was subjected to 2-D-PAGE. The sample was denatured for the first dimension in buffer without DTE to avoid the dissociation of ANP-protein complexes. DTE was also omitted in all the subsequent steps. As shown in Fig. 2, the gel autoradiography revealed the presence of many ANP-bound radioactive spots in the range 40$200 \mathrm{kDa}$ and pI 3-8. The identification of the proteins present in the radioactive spots was performed by MALDI-TOF mass spectrometry. Orosomucoid, $\alpha-1$ antitrypsin, a dimeric form of apo A-I, many albumin isoforms and apo A-IV-TTR complex were identified.

\section{Discussion}

In a previous work (Maioli et al. 2000), we hypothesized that ANP binds to plasma proteins and that this interaction may maintain ANP in a soluble form, thus preventing its aggregation and therefore suppressing its fibril-forming propensity. Recently (Torricelli et al. 2004), we reported that a dimeric form of apo A-I, the apolipoprotein most represented in HDL, is able to bind ANP.

In the present work, by using a different approach, we confirmed that ANP binds to the apo A-I dimer. This result strengthened the idea that apo A-I is one of the most important factors in preventing the amyloid disease. However, the results obtained by binding-inhibition experiments showed that anti-apo A-I antibodies could not either inhibit binding in solution or change the electrophoretic profile of protein-bound ANP (Fig. 1). This finding suggested that apo A-I is not 


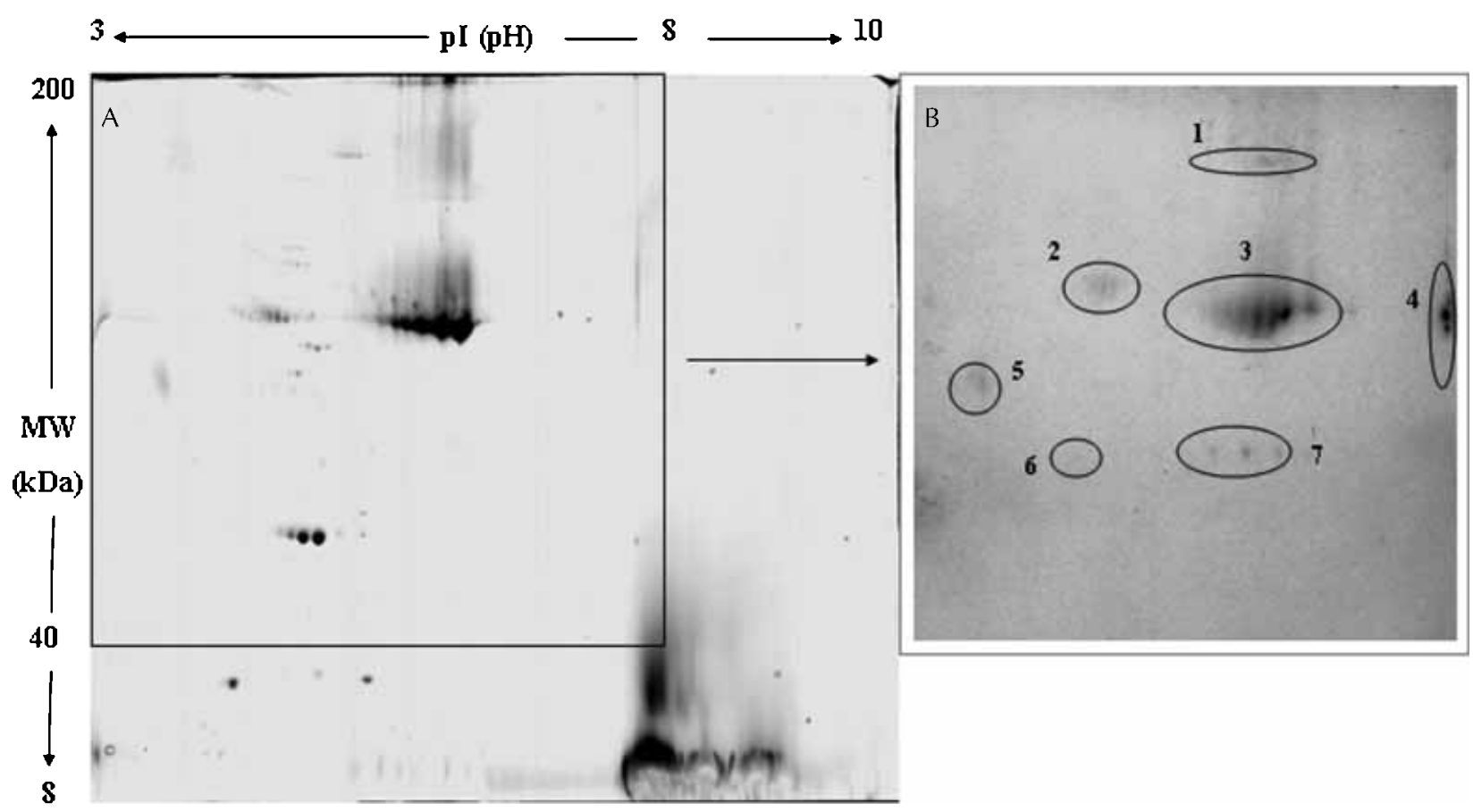

Figure 2 Representative two-dimensional gel (2-D) electrophoresis and autoradiography patterns of big $\alpha$-I ${ }^{125}$-hANP. After incubation of $\alpha-\left.\right|^{125}$-hANP and whole plasma big $\alpha-\left.\right|^{125}$-hANP was applied on the 2-D gel under non-reducing conditions. The gel was stained with Coomassie brilliant blue, dried and subjected to autoradiography.

(A) Electrophoretogram; (B) region of the gel where $\alpha-I^{125}$-hANP-binding proteins were revealed by autoradiography. 1, albumin dimer; 2, $\alpha-1$ antitrypsin; 3, albumin; 4, albumin isoform; 5, orosomucoid; 6, apo A-I dimer; 7, apo A-IV-TTR complex. All experiments were done in duplicate on at least three independent occasions.

the only protein involved in ANP binding. Indeed, plasma contains at least four times greater amyloid inhibitory activity than HDL. These results prompted us to search for additional proteins able to interact with the peptides that were identified in the present work.

We found that ANP binds to a complex formed by TTR and apo A-IV. Interestingly, ANP binds to the TTR-apo A-IV complex, as well as the apo A-I dimer, but not to the isolated proteins. ANP/apo A-I and ANP/apo A-IV-TTR interactions probably depend on the three-dimensional conformation assumed by the binding proteins in the complexes. This peculiar ANP behaviour strongly differs from that of $\mathrm{A} \beta$, which binds to the monomeric forms of apo A-I, TTR and apo A-IV (Shuvaev \& Siest 1996, Koudinov et al. 1998, Tsuzuki et al. 2000).

The presence of a TTR-apo A-IV complex in human plasma is a novel finding, never reported previously. However, a recent work (Bergstrom et al. 2001) reports a codeposition of apo A-IV and wild-type TTR in amyloid deposits isolated from the heart of a patient with senile systemic amyloidosis, thus indicating a kind of interaction between these two proteins.

We have found that the majority of iodinated ANP added to plasma was bound to albumin. Albumin is the most abundant protein in plasma. The albumin involvement in ANP binding is not surprising. In fact, albumin has been reported to be one of the major $A \beta$-binding proteins in the circulation and one of the most potent inhibitors of A $\beta$ polymerization (Bohrmann et al. 1999). We have shown that ANP binds several albumin isoforms, including an albumin form having a higher molecular mass corresponding to an albumin dimer. Its molecular mass approximately corresponds to the $>94 \mathrm{kDa}$ band shown in the monodimensional gel (Fig. 2). While the albumin dimer is reported to be present in the circulation (Atmeh \& Shabsoug 1997), nothing is known about the minor isoform at very basic pI identified in our study by labelled ANP. Our present result apparently conflicts with our early studies showing 
no additive ANP binding to HSA-enriched plasma (Maioli et al. 2000). However, the presence in normal plasma of several albumin isoforms with ANP-binding properties might reconcile present and previous results. In fact, the highly purified HSA used to enrich plasma probably did not contain all the albumin species present in the bloodstream. Moreover, as we can see from the present work, albumin works in concert with many other proteins in the ANP-binding process.

In fact, some ANP was also bound to $\alpha-1$ antitrypsin, a serine-protease inhibitor which is known to be implicated in amyloid disease at several levels. For example, it is one of the plasma factors able to bind A $\beta$ (Bohrmann et al. 1999) and can disaggregate light-chain-derived amyloid fibrils in vitro (Eriksson et al. 1995). Moreover, certain C-terminal fragments of $\alpha-1$ antitrypsin are able to form amyloid fibrils in vitro, a process that can be regulated by the intact $\alpha-1$ antitrypsin molecule (Janciauskiene et al. 1995).

Finally, orosomucoid, or acid $\alpha-1$ glycoprotein, is another ANP-binding protein. An involvement of this protein in the amyloid disease has never been reported before. Thus, our result extends the list of factors protective against amyloid aggregation. It would be of interest to check the ability of this protein to bind amyloidogenic peptides other than ANP.

The existence of various physiological ligands for ANP and $A \beta$ supports the idea that the human organism has been provided with various protective agents, common to various amyloidogenic peptides, able to inhibit fibril formation and thus amyloid disease.

The advantage of the existence of multiple sequestering proteins could allow us to avoid the saturation of the protective mechanism and, at the same time, to overcome the risk resulting from the bad functioning or the deficiency of one component of the system.

An intriguing scenario emerges from this perspective. Polypeptides such as TTR, apo A-I, apo A-IV and $\alpha-1$ antitrypsin are 'two-faced' molecules. In fact, these human proteins are known to bind A $\beta$ (Koudinov et al. 1994, Bohrmann et al. 1999, Tsuzuki et al. 2000) or ANP (present paper), probably preventing fibrillar aggregation. At the same time, all these factors exhibit the intrinsic potential to form amyloid deposits (Sletten et al. 1980, Pitkanen et al. 1984, Janciauskiene et al. 1995,
McCarthy \& Kasper 1998, Bergstrom et al. 2001, Andreola et al. 2003).

This dual feature leads to paradoxical situations. Considering, for example, the interaction between apo A-I and $A \beta$, one could infer that the higher the apo A-I amount, the lower is the risk of amyloidosis development by $\mathrm{A} \beta$. However, this is not the case, since high apo A-I concentration might favour self-assembly in amyloid fibrils of apo A-I monomers, rather than inhibit $\mathrm{A} \beta$ polymerization. Then, how can we distinguish the apo A-I protective role from the risk of amyloid formation? The answer to this enigma probably lies in the relative local concentration of both peptides, as speculated by McLaurin et al. 2000.

\section{Acknowledgement}

This work was partially supported by a grant from the University of Siena, Italy (Piano di Ateneo per la Ricerca, Esercizio 2003, Quota per Servizi, Area delle Scienze Biomediche e Mediche).

\section{References}

Andreola A, Bellotti V, Giorgetti S, Mangione P, Obici L, Stoppini M, Torres J, Monzani E, Merlini G \& Sunde M 2003 Conformational switching and fibrillogenesis in the amyloidogenic fragment of apolipoprotein A-I. Fournal of Biological Chemistry 278 2444-2451.

Atmeh RF \& Shabsoug B 1997 Detection and semiquantitation of albumin forms in fresh human plasma separated on gradient polyacrylamide gel by means of electroblotting on agarose gel matrix. Electrophoresis 18 2055-2058.

Bergstrom J, Murphy C, Eulitz M, Weiss DT, Westermark GT, Solomon A \& Westermark P 2001 Codeposition of apolipoprotein A-IV and transthyretin in senile systemic (ATTR) amyloidosis. Biochemical and Biophysical Research Communications 285 903-908.

Bohrmann B, Tjernberg L, Kuner P, Poli S, Levet-Trafit B, Naslund J, Richards G, Huber W, Dobeli H \& Nordstedt G 1999 Endogenous proteins controlling amyloid beta-peptide polymerisation. Possible implications for beta-amyloid formation in the central nervous system and in peripheral tissues. Fournal of Biological Chemistry 247 15990-15995.

Eriksson S, Janciauskiene S \& Merlini G 1995 The putative role of alpha-1-antitrypsin in the disaggregation of amyloid lambda fibrils. fournal of Internal Medicine 237 143-149.

Janciauskiene S, Carlemalm E \& Eriksson S 1995 In vitro fibril formation from alpha 1-antitrypsin-derived C-terminal peptides. Biological Chemistry 376 415-423.

Jarrett JT, Berger EP \& Lansbury PT Jr 1993 The carboxy terminus of the beta amyloid protein is critical for the seeding of amyloid formation: implications for the pathogenesis of Alzheimer's disease. Biochemistry 32 4693-4697.

Johansson B, Wernstedt C \& Westermark P 1987 Atrial natriuretic peptide deposited as atrial amyloid fibrils. Biochemical and Biophysical Research Communications 148 1087-1092. 
Johansson B \& Westermark P 1990 The relation of atrial natriuretic factor to isolated atrial amyloid. Experimental and Molecular Pathology 52 266-278.

Koudinov A, Matsubara E, Frangione B \& Ghiso J 1994 The soluble form of Alzheimer's amyloid beta protein is complexed to high density lipoprotein 3 and very high density lipoprotein in normal human plasma. Biochemical and Biophysical Research Communications 205 1164-1171.

Koudinov AR, Berezov TT, Kumar A \& Koudinova NV 1998 Alzheimer's amyloid $\beta$ interaction with normal human plasma high density lipoprotein: association with apolipoprotein and lipids. Clinica Chimica Acta 270 75-84.

Lachmann HJ \& Hawkins PN 2003 Novel pharmacological strategies in amyloidosis. Nephron Clinical Practice 94 c85-88.

Looi LM 1993 Isolated atrial amyloidosis: a clinicopathologic study indicating increased prevalence in chronic heart disease. Human Pathology 24 602-607.

Maioli E, Torricelli C, Santucci A \& Pacini A 2000 Molecular assembly of endogenous and synthetic big atrial natriuretic peptide (ANP) and its amyloidogenic implications. Biochimica et Biophysica Acta 1500 31-40.

Maioli E, Torricelli C, Santucci A, Martelli P \& Pacini A 2001 Plasma factors controlling atrial natriuretic peptide (ANP) aggregation: role of lipoproteins. Biochimica et Biophysica Acta 1536 123-132.

McCarthy RE 3rd \& Kasper EK 1998 A review of the amyloidoses that infiltrate the heart. Clinical Cardiology 21 547-552.

McLaurin J, Yang D, Yip CM \& Fraser PE 2000 Review: modulating factors in amyloid-beta fibril formation. Fournal of Structural Biology 130 259-270.

Merlini G \& Bellotti V 2003 Molecular mechanisms of amyloidosis. New England Fournal of Medicine 349 583-596.

Pitkanen P, Westermark P \& Cornwell GG 3rd 1984 Senile systemic amyloidosis. American Fournal of Pathology 117 391-399.

Rocken C, Peters B, Juenemann G, Saeger W, Klein HU, Huth C, Roessner A \& Goette A 2002 Atrial amyloidosis: an arrhythmogenic substrate for persistent atrial fibrillation. Circulation 106 2091-2097.
Shuvaev VV \& Siest G 1996 Interaction between human amphipathic apolipoproteins and amyloid beta-peptide: surface plasmon resonance studies. FEBS Letters 383 9-12.

Sletten K, Westermark P \& Natvig JB 1980 Senile cardiac amyloid is related to prealbumin. Scandinavian Fournal of Immunology 12 503-506.

Sousa MM, Berglund L \& Saraiva MJ 2000 Transthyretin in high density lipoproteins: association with apolipoprotein A-I. Fournal of Lipid Research 41 58-65.

Takahashi M, Hoshii Y, Kawano H, Gondo T, Yokota T, Okabayashi H, Shimada I \& Ishihara T 1998 Ultrastructural evidence for the formation of amyloid fibrils within cardiomyocytes in isolated atrial amyloid. Amyloid 5 35-42.

Talamo F, D'Ambrosio C, Arena S, Del Vecchio P, Ledda L, Zehender G, Ferrara L \& Scaloni A 2003 Proteins from bovine tissues and biological fluids: defining a reference electrophoresis map for liver, kidney, muscle, plasma and red blood cells. Proteomics 3 440-460.

Tan SY \& Pepys MB 1994 Amyloidosis. Histopathology 25 403-414.

Torricelli C, Capurro E, Santucci A, Paffetti A, D'Ambrosio C, Scaloni A, Maioli E \& Pacini A 2004 Small HDL form via Apo A-I a complex with atrial natriuretic peptide. Biochemical and Biophysical Research Communications 315 16-21.

Trabalzini L, Paffetti A, Scaloni A, Talamo F, Ferro E, Coratza G, Bovalini L, Lusini P, Martelli P \& Santucci A 2003 Proteomic response to physiological fermentation stresses in a wild-type wine strain of Saccharomyces cerevisiae. Biochemical fournal 370 35-46.

Tsuzuki K, Fukatsu R, Yamaguchi H, Tateno M, Imai K, Fujii N \& Yamauchi T 2000 Transthyretin binds amyloid beta peptides, Abetal-42 and Abetal-40, to form complex in the autopsied human kidney - possible role of transthyretin for abeta sequestration. Neuroscience Letters 281 171-174.

\section{Received in final form 12 February 2004} Accepted 7 July 2004 Made available online as an Accepted Preprint 22 July 2004 